\title{
Search for Gamma-Ray Emission from the Nova-like Variable AE Aquarii Using the Fermi-LAT Pass 8 Data Archive
}

\author{
S.T. Madzime ${ }^{* \dagger}$ \\ Department of Physics, University of the Free State, Bloemfontein, South Africa \\ E-mail: madizimesteufs.ac.za

\section{P.J. Meintjes} \\ Department of Physics, University of the Free State, Bloemfontein, South Africa \\ E-mail: MeintjPJ@ufs.ac.za

\section{K.K. Singh} \\ Department of Physics, University of the Free State, Bloemfontein, South Africa \\ Astrophysical Sciences Division, Bhabha Atomic Research Centre, Mumbai 400085, India \\ E-mail: SinghKK@ufs.ac.za
}

\section{H.J. van Heerden}

Department of Physics, University of the Free State, Bloemfontein, South Africa E-mail: VanHeerden@ufs.ac.za

We present the preliminary results of the search for gamma-ray emission from the novalike variable AE Aquarii using the upgraded Fermi-LAT Pass 8 data set. A previous study using the Fermi-LAT Pass 7 data pipeline showed indications of a low-level, but consistent, pulsed emission at a period of $16.54 \mathrm{~s}$, which is the first harmonic of the $33.08 \mathrm{~s}$ spin period of the white dwarf. Here we report our findings of a follow-up study utilizing the Pass 8 data, which uses an improved galactic diffuse gamma-ray emission model as well as more inclusive selection criteria. Thus far no clear detection of any significant gamma-ray excess from AE Aqr in the Fermi-LAT energy range has been made. However, there are some energy bins with significance just above $2 \sigma$. This significance value is below the Fermi-LAT detection limit that signifies a clear 4-5 $\sigma$ detection and can only be considered as a $2 \sigma$ upper-limit. The corresponding upper limit of the integrated energy flux above $100 \mathrm{MeV}$ is $F_{\gamma} \sim 4 \times 10^{-13} \mathrm{erg} \mathrm{cm}^{-2} \mathrm{~s}^{-1}$ which is determined at the $99.995 \%$ confidence level. However, indications of low-level pulsed modulation is found at, or close to, the $33.08 \mathrm{~s}$ spin period of the white dwarf in a few two-week data sections with higher than average TS values, which is promising for possible future follow-up studies with the Cherenkov Telescope Array.

7th Annual Conference on High Energy Astrophysics in Southern Africa - HEASA2019

28 - 30 August 2019

Swakopmund, Namibia

* Speaker.

${ }^{\dagger}$ The authors thank the organizers for the invitation to present this work at this conference. They also want to extend their gratitude to the University of the Free State postgraduate school, SA-GAMMA, physics department and National Research Foundation (NRF) for assistance. The Fermi-LAT analysis was performed utilizing the University of the Free State High-Performance Computing Unit (HPC). 


\section{Introduction}

AE Aquarii (AE Aqr) is a novalike cataclysmic variable, a close binary which consists of a fast rotating magnetized white dwarf, orbiting a late-type main sequence companion (K4-5) star, with an orbital period of $9.88 \mathrm{hrs}$ (e.g., $[1,2,3])$. The white dwarf is highly magnetic ( $\sim \mathrm{MG})$ and rotates with a period of $\sim 33 \mathrm{~s}$ [4]. AE Aqr exhibits novalike characteristics in optical wavelengths with a visual magnitude varying between $m_{V}=10$ and $m_{V}=12$ [5]. It shows highly transient multi-wavelength emission characteristics as revealed by several studies: optical (e.g., [4, 3, 6]), radio (e.g., [7, 8, 9]), $\mathrm{X}$-rays (e.g., [10,11]) and possibly in $\mathrm{TeV}$ gamma-rays energy bands (e.g., $[12,13,14])$.

The peculiar multi-wavelength emission from AE Aqr is intimately tied to the fast rotating magnetosphere of the compact object and propeller ejection of matter from the system. The propeller mechanism in AE Aqr is the result of the rotational velocity of the white dwarf's magnetosphere exceeding the Keplerian velocity of the mass flow stream from the secondary star [15], resulting in centrifugal expulsion of material from the binary system. Two models can explain the mechanism driving the propeller ejection of matter from the system of AE Aqr. The models are magnetic propulsion of diamagnetic blobs as they interact with the fast rotating magnetosphere (e.g., $[16,17])$, and mixing of the magnetic field through Kelvin-Helmholtz instabilities resulting in magnetized blobs being ejected from the system (e.g., $[18,19])$. The propeller effect will result in braking of the spinning white dwarf at a rate of $\dot{P} \sim 6 \times 10^{-14} \mathrm{~s} \mathrm{~s}^{-1}$. Since the spin-down power exceeds the accretion luminosity, a fraction of the spin-down power provides a substantial reservoir of energy that may be channeled into particle acceleration to high energies and non-thermal emission (e.g., [20,21]). It has been shown that the white dwarf in AE Aqr could accelerate electrons and protons to energies of the order of several $\mathrm{TeV}\left(1 \mathrm{TeV}=10^{12} \mathrm{eV}\right)[15]$, which could produce gamma-rays through a leptonic channel (inverse Compton) or a hadronic channel i.e., neutral pion production and decay to gamma-rays (e.g., [22, 23, 24, 25]). Evidence of particle acceleration in AE Aqr is the detection of non-thermal radio emission [8] and the Suzaku detection of a pulsed hard $\mathrm{X}$-ray component above $10 \mathrm{keV}$ which shows a non-thermal power law spectrum with a photon index of $\Gamma=1.2$. This detection could be an indication of synchrotron radiation from relativistic electrons in the white dwarf magnetosphere (e.g., [11, 15]).

\section{Fermi-LAT Data Reduction and Analysis}

The archival gamma-ray data from Fermi Large Area Telescope (Fermi-LAT) collected between 4 Aug 2008 to 30 Nov 2018 on AE Aqr were considered for this study. The data set comprises of all Fermi-LAT events and spacecraft data for detailed analysis. The analysis of the Fermi-LAT data were performed using Fermi Science Tools software packages (v11r5p3). We used the P8R3_SOURCE_V2 set of response functions and selected corresponding source-class events (evclass $=128$ ) and front+back events type (evtype $=3$ ). In this analysis, photons were selected from a radius of 10 degrees Region Of Interest (ROI), centered at the coordinates of AE Aqr $(\mathrm{RA}=310.038, \mathrm{DEC}=-0.8708$ epoch $\mathrm{J} 2000)$. The zenith angle cut was set at 90 degrees to avoid contamination by photons produced from cosmic-ray interactions with the atmosphere. Binned maximum $-\log ($ likelihood) analysis was performed in the energy range $0.1-500 \mathrm{GeV}$ [26]. All 
sources within 20 degrees of ROI, the instrumental background, and the diffuse galactic and extragalactic isotropic emission model gll_iem_v06.fits [27] as well as the source of interest were included in the spectral model file. The analysis of AE Aqr was performed utilizing the simplest spectral model for non-thermal emission (i.e., a Power Law).

$$
\frac{d N}{d E}=N_{0}\left[\frac{-E}{E_{0}}\right]^{-\Gamma}
$$

where $\Gamma$ is the photon index which controls the hardness of the source, the scale $N_{0}$ which is the normalization factor and the pivot energy $E_{0}$, which scales the energy. These parameters and other parameters in the spectral-file model were optimized and fitted using the maximum $-\log$ (likelihood) technique, [26]. The significance of a detection of AE Aqr was estimated using the test statistic (TS), which is the ratio of $-\log$ (likelihood) with and without source. The statistical significance of the results is expressed as $\sigma \approx \sqrt{T S}$ [26].

The search for a periodic modulation of gamma-rays from the region of AE Aqr was performed using gtpsearch and gtpphase routines of Fermi Science Tools software packages (v10r0p5). The entire data set was divided into 14-days sections and the sections with higher than average TS value were selected for periodic analysis. Python scripts were developed to use these tools effectively. Both gtpsearch and gtpphase routine utilize the spacecraft file and coordinates of the source of interest to perform the barycentric correction of each photon's time of arrival. The spin ephemeris of AE Aqr used in this analysis were adopted from Jian et al., [28].
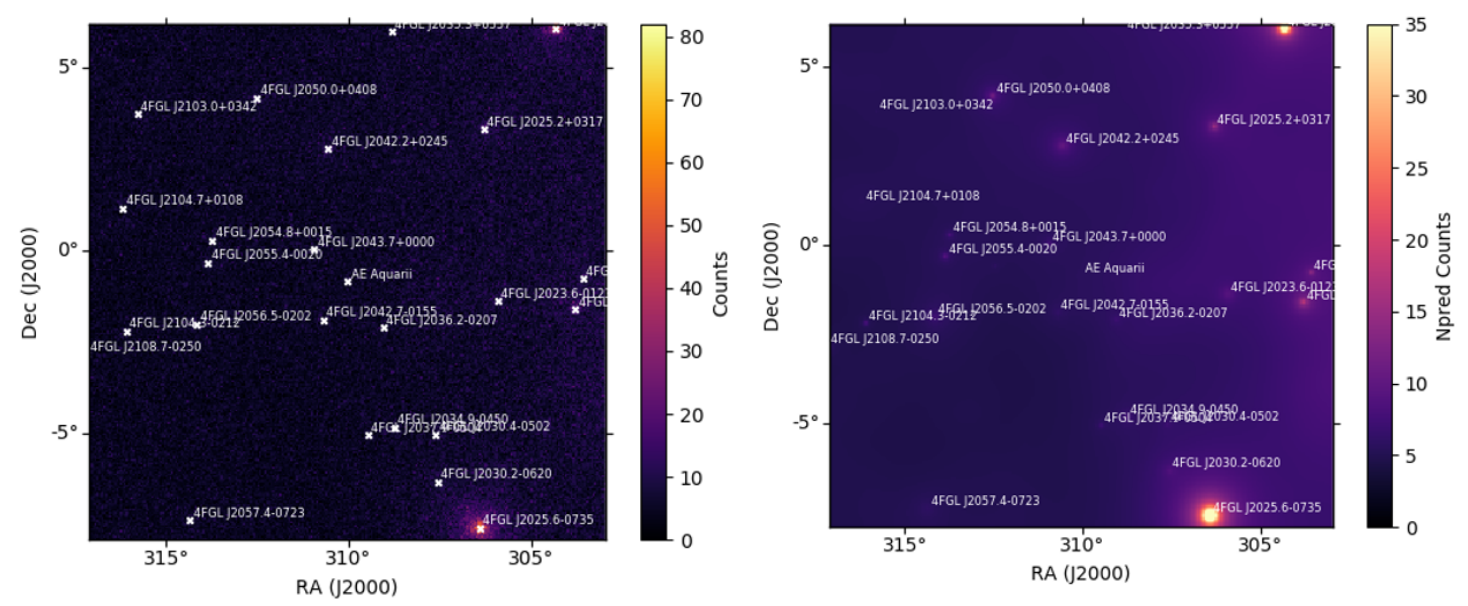

Figure 1: Counts map (left) and model map (right). The counts map is the cumulative data of observed counts at a given grid location. A closer inspection of the counts map generated for ten years of data shows no extremely bright sources in the ROI. The background around the region of AE Aqr is brighter than the source of interest. It can be seen from the model map that there is no sign of a point source at the region of AE Aqr. The predicted counts of AE Aqr after accounting for background emission is negligible, as shown in the model map.

\section{Results}

The binned analysis of Fermi-LAT data of AE Aqr over the last decade revealed no statistically significant gamma-ray excess in the energy range of $0.1-500 \mathrm{GeV}$. For a point source 
detection at the 4-5 sigma confidence level, an average TS value between 16-25 is required [26]. The average TS for AE Aqr in the energy interval $0.1-500 \mathrm{GeV}$ signifies a clear non-detection at the 4-5 sigma level and therefore only a $2 \sigma$ upper limit is determined at a flux level of $\sim 2 \times 10^{-9} \mathrm{ph} \mathrm{cm}^{-2} \mathrm{~s}^{-1}$ above $100 \mathrm{MeV}$ at the $99.995 \%$ confidence level. This corresponds to an integrated energy flux above $100 \mathrm{MeV}$ of $F_{\gamma} \sim 4 \times 10^{-13} \mathrm{erg} \mathrm{cm}^{-2} \mathrm{~s}^{-1}$.

We verified our results' accuracy by comparing the raw data map to the likelihood model map (see Figure 1). Counts map (map of raw data) is produced from filtered raw data with Good Time Intervals (GTIs') applied. On the other hand, the model map is a diagnostic plot that shows each source's predicted counts in the fit. The difference between the model map and the counts is the residual, which is usually flat for a good fit. There is no clear indication of a point source at the region of AE Aqr, as shown by both the model map and the counts map. The predicted counts in the model map and the observed counts in the counts map appear to be comparable. Even after accounting for the background emission, the gamma-ray flux from the region of AE Aqr is at a low level, as can be seen from the model map.

We also investigated the spectral behaviour of the low-level gamma-rays. We conducted the investigation by generating the spectral flux points using 30 energy bins per decade to see whether the model agrees with the data and check which energy bins contribute more to the obtained significance of gamma-ray emission from AE Aqr. Unfortunately, no energy bin was found with flux points with significance above $3 \sigma$. Thus some energy bins with flux points that have significance above $2 \sigma$ were considered flux points, not upper limits. On the left-hand side of Figure 2 is a spectral energy distribution (SED) plot fitted with a power law with a spectral index of $\Gamma \sim 2$. The right-hand side of Figure 2 represents a broadband SED, plotted with $2 \sigma$ flux points from this study, published multi-wavelength archival results (see e.g., [15]), and Cherenkov Telescope Arrays (CTA), and Fermi-LAT sensitivity curves.
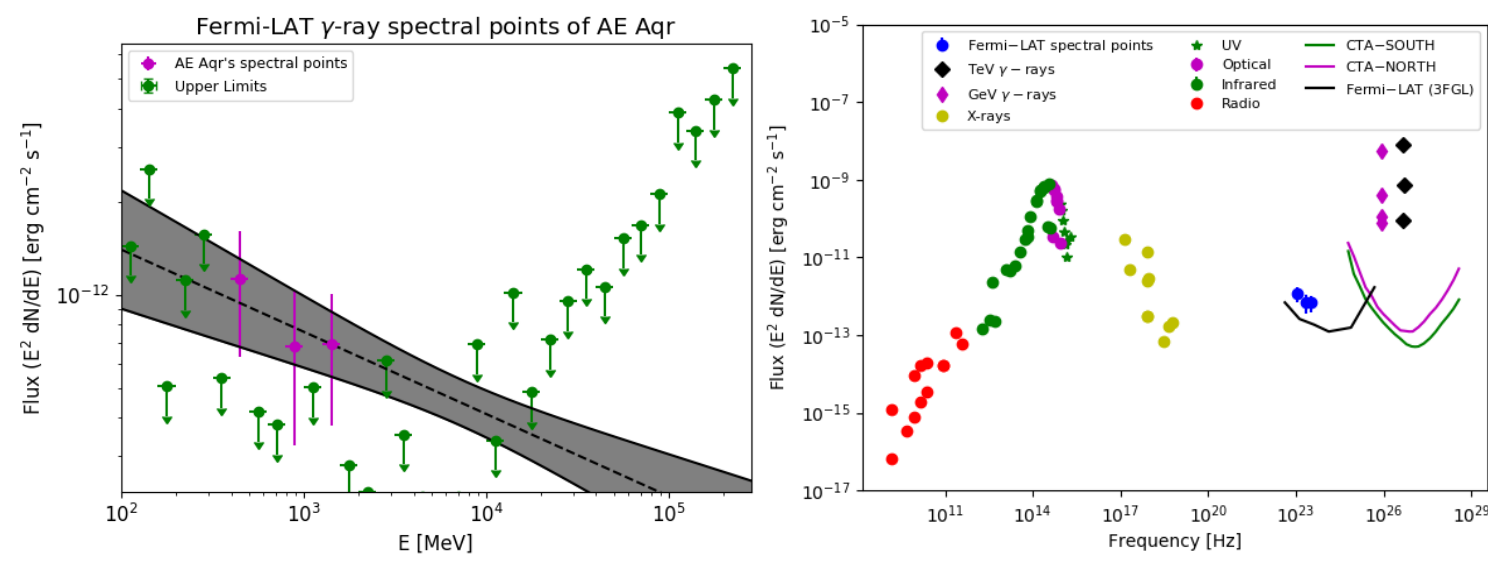

Figure 2: The left panel is showing the Fermi-LAT flux points at $(2 \sigma)$ and upper limits from bins with low TS value. The fit through the $2 \sigma$ flux points is a power law with a photon index $\Gamma \sim 2$. The right panel is showing the broadband SED of AE Aqr from radio to very high energy emission (see e.g., [15]) including CTA, and Fermi-LAT sensitivity curves. The gamma-ray flux levels around $100 \mathrm{GeV}$ (purple diamonds) are burst-like events detected by the Durham group [14] and the TeV gamma-ray flux levels (black diamonds) were transient burst-like events detected with the Nooitgedacht Mk I Cherenkov telescope[13]. Both groups reported these events contemporaneously in the late 1980s and early 1990s, see Oruru and Meintjes [15] (and references therein) for a discussion. 
To search for pulsed gamma-ray from AE Aqr the Rayleigh statistic was utilized as part of the Fermi-LAT science tools over the energy range $0.1-500 \mathrm{GeV}$. The data set was divided into 14-day sections and the analysis was restricted to those sections with highest TS value. We also extended our search by employing narrower energy bins, but all investigations were conducted with $10^{\circ}$ radius of the ROIs. The maximum Rayleigh test statistic $\left(2 n R^{2}\right)$ [29] obtained in this analysis is 13.8, which corresponds to a probability for chance occurrence of $\operatorname{Pr}=e^{-n R^{2}} \approx 10^{-3}$ [29]. The power occurs at or near the rotational period of the white dwarf in AE Aqr (see Figure 3). However, the nature of the data and the sparsity of the events makes it impossible to assign any real statistical significance to these results.
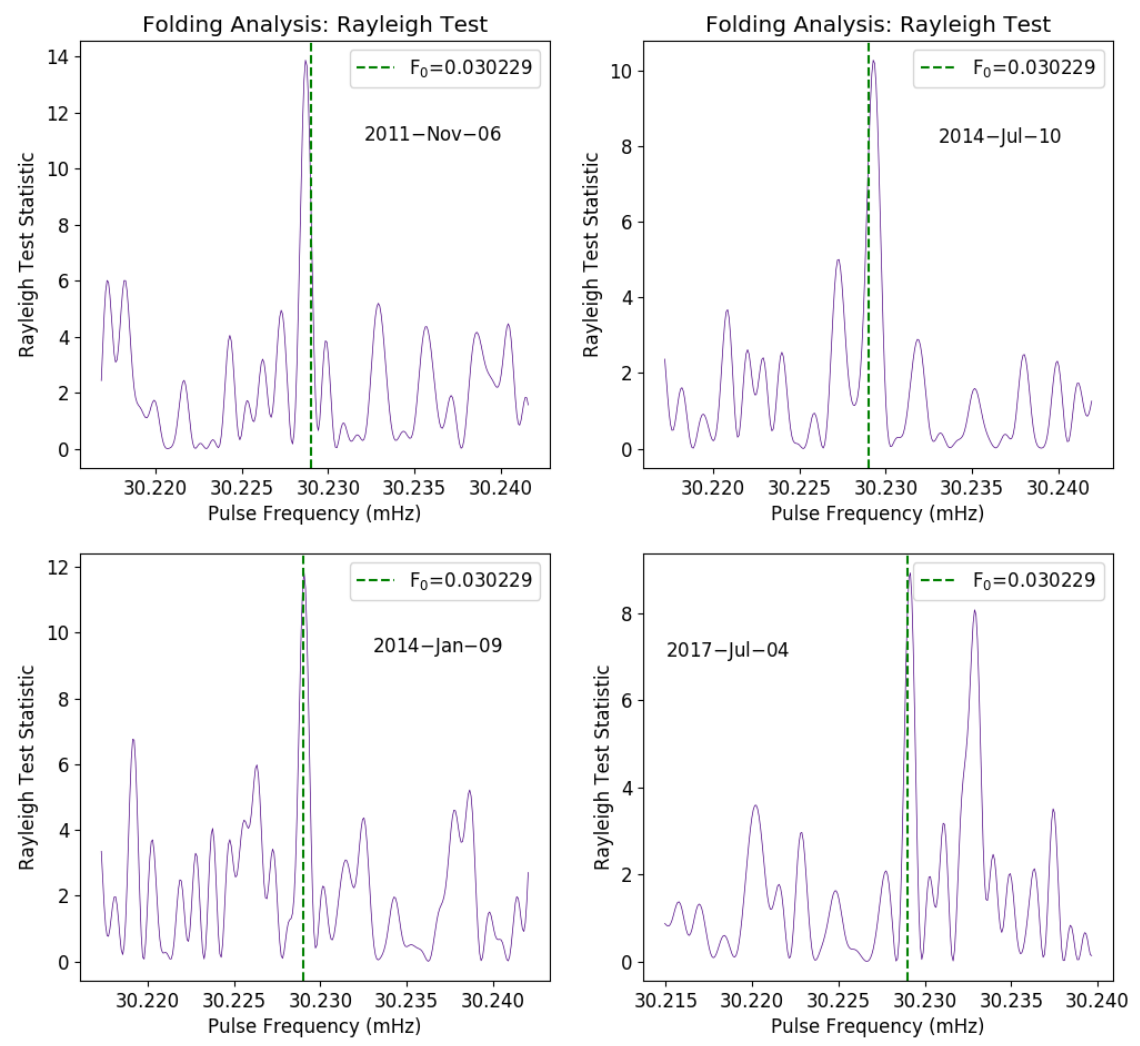

Figure 3: The folding analysis conducted using the Rayleigh test revealed low-level pulsed modulation at or near the $\sim 33.08 \mathrm{~s}$ white dwarf rotational period, indicated by the dashed line.

\section{Conclusion}

This study resulted in no significant detection of gamma-ray emission from the region of $\mathrm{AE}$ Aqr above the $2 \sigma$ detection threshold of Fermi-LAT. Since the overall significance of the source is low over the entire energy range, we investigated energy bins that contributed more to the possible emission. In our results, we observed that in most of the energy bins, the source is insignificant except for a few energy bins with significance that barely exceeds $2 \sigma$. The energy range with $2 \sigma$ significance is above the energy range that is most affected by the background gamma-ray contamination. However, it should be mentioned that the background in this region is brighter 
than most sources, as seen in the counts map (see Figure 1). Even the model map shows that the number of predicted counts for each source still results in some sources with negligible counts (see Figure 1).

Previous studies in the late 1980s and early 1990s suggested possible aperiodic burst-like high energy gamma-ray (VHE; TeV) emission from AE Aquarii. The unpredictable nature of these burst-like events introduces severe challenges in detecting possible high energy gamma-ray emission from AE Aquarii with the Fermi-LAT telescope, which has poor angular resolution and sensitivity compared to current ground-based Atmospheric Cherenkov Telescope Arrays. Therefore future studies with instruments like CTA may reveal more conclusive results.

\section{References}

[1] A. H. Joy, Spectroscopic observations of AE Aquarii., The Astrophysical Journal 120 (1954) 377.

[2] J. A. Crawford and R. P. Kraft, An intrepretation of AE Aquarii., The Astrophysical Journal 123 (1956) 44.

[3] G. Chincarini and M. F. Walker, Image tube spectroscopic studies of rapid variables. iv-spectroscopic and photometric observations of AE Aquarii, Astronomy and Astrophysics 104 (1981) 24.

[4] J. Patterson, Rapid oscillations in cataclysmic variables. iii-an oblique rotator in an AE Aquarii, The Astrophysical Journal 234 (1979) 978.

[5] E. Zinner, Mitteilungen über veränderliche und verdächtige Sterne, Astronomische Nachrichten 265 (1938) 345.

[6] M. Eracleous and K. Horne, The speedy magnetic propeller in the cataclysmic variable AE Aquarii, The Astrophysical Journal 471 (1996) 427.

[7] J. A. Bookbinder and D. Q. Lamb, Discovery of radio emission from AE Aquarii, The Astrophysical Journal 323 (1987) L131.

[8] T. S. Bastian, G. A. Dulk and G. Chanmugam, Radio flares from AE Aquarii-a low-power analog to Cygnus X-3?, The Astrophysical Journal 324 (1988) 431.

[9] M. Abada-Simon, A. Lecacheux, T. S. Bastian, J. A. Bookbinder and G. A. Dulk, The spectrum and variability of radio emission from AE Aquarii, The Astrophysical Journal 406 (1993) 692.

[10] J. Patterson and C. Price, Rapid oscillations in cataclysmic variables. v-h2252-035, a single-sideband X-ray and optical pulsar, The Astrophysical Journal 243 (1981) L83.

[11] Y. Terada, M. Ishida, K. Mukai, T. Dotani, K. Makishima, S. Naik et al., Possible Suzaku detection of non-thermal X-ray signals from a rotating magnetized white dwarf, Advances in Space Research 41 (2008) 512.

[12] P. J. Meintjes, Simultaneous optical and TeV gamma-ray observations of the cataclysmic variable AE Aquarii, Thesis, Potchefstroom Univ. for CHE (1992) .

[13] P. J. Meintjes, O. C. De Jager, B. C. Raubenheimer, H. I. Nel, A. R. North, D. A. H. Buckley et al., Simultaneous optical and TeV gamma-ray observations of the cataclysmic variable AE Aquarii, The Astrophysical Journal 434 (1994) 292.

[14] P. Chadwick, J. Dickinson, M. Dickinson, N. Dipper, J. Holder, T. McComb et al., A burst of pulsed VHE gamma rays from AE Aquarii, Astroparticle Physics 4 (1995) 99. 
[15] B. Oruru and P. J. Meintjes, $X$-ray characteristics and the spectral energy distribution of AE Aquarii, Monthly Notices of the Royal Astronomical Society 421 (2012) 1557.

[16] G. A. Wynn, A. R. King and K. Horne, A magnetic propeller in the cataclysmic variable AE Aquarii, Monthly Notices of the Royal Astronomical Society 286 (1997) 436.

[17] P. J. Meintjes and L. A. Venter, The diamagnetic blob propeller in AE Aquarii and non-thermal radio to mid-infrared emission, Monthly Notices of the Royal Astronomical Society 360 (2005) 573.

[18] P. Meintjes and L. Venter, Modelling the continuous radio outbursts in AE Aquarii, Monthly Notices of the Royal Astronomical Society 341 (2003) 891.

[19] L. A. Venter and P. J. Meintjes, Kelvin-Helmholtz driven propeller in AE Aquarii: A unified model for thermal and non-thermal flares, Monthly Notices of the Royal Astronomical Society 366 (2006) 557.

[20] O. De Jager, P. Meintjes, D. O'Donoghue and E. Robinson, The discovery of a brake on the white dwarf in AE Aquarii, Monthly Notices of the Royal Astronomical Society 267 (1994) 577.

[21] P. Meintjes and O. De Jager, Propeller spin-down and the non-thermal emission from AE Aquarii, Monthly Notices of the Royal Astronomical Society 311 (2000) 611.

[22] P. Meintjes, Cosmic accelerators: New surprises in the CTA era, in Frontier Research in Astrophysics-II, vol. 269, p. 031, SISSA Medialab, 2017.

[23] E. Lorenz and R. Wagner, Very-high energy gamma-ray astronomy, The European Physical Journal H 37 (2012) 459.

[24] Fermi-LAT Collaboration et al., Fermi establishes classical novae as a distinct class of gamma-ray sources, Science 345 (2014) 554.

[25] B. D. Metzger, T. Finzell, I. Vurm, R. Hascoet, A. M. Beloborodov and L. Chomiuk, Gamma-ray novae as probes of relativistic particle acceleration at non-relativistic shocks, Monthly Notices of the Royal Astronomical Society 450 (2015) 2739.

[26] J. R. Mattox, D. Bertsch, J. Chiang, B. Dingus, S. Digel, J. Esposito et al., The likelihood analysis of EGRET data, The Astrophysical Journal 461 (1996) 396.

[27] F. Acero, M. Ackermann, M. Ajello, A. Albert, L. Baldini, J. Ballet et al., Development of the model of galactic interstellar emission for standard point-source analysis of Fermi Large Area Telescope data, The Astrophysical Journal 223 (2016) 26.

[28] J. Li, D. F. Torres, N. Rea, E. de Ona Wilhelmi, A. Papitto, X. Hou et al., Search for gamma-ray emission from AE Aquarii with seven years of FERMI-LAT observations, The Astrophysical Journal 832 (2016) 35.

[29] K. Mardia, Statistics of directional data. Academic Press, New York, 1972. 